\title{
Organogels from a Vitamin C-Based Surfactant
}

\section{Pierandrea Lo Nostro, ${ }^{*}, \dagger$ Roland Ramsch,${ }^{\dagger}$ Emiliano Fratini, ${ }^{\dagger}$ Marco Lagi, ${ }^{\dagger}$ Francesca Ridi, ${ }^{\dagger}$ Emiliano Carretti, ${ }^{\dagger}$ Moira Ambrosi, ${ }^{\dagger}$ Barry W. Ninham,${ }^{\dagger}$ and Piero Baglioni ${ }^{\dagger}$}

Department of Chemistry and CSGI, University of Florence, 50019 Sesto Fiorentino (Firenze), Italy, and Department of Applied Mathematics, Research School of Physical Sciences and Engineering, Institute of Advanced Studies, Australian National University, Canberra, Australia 0200

Received: April 18, 2007; In Final Form: July 27, 2007

\begin{abstract}
A new double chained surfactant, 2-octyl-dodecanoyl-6-O-ascorbic acid (8ASC10), with a L-ascorbic acid unit as the polar headgroup was synthesized for the first time. The behavior of the compound in the dry solid state has been characterized through DSC, XRD, and SAXS measurements. The surfactant forms stable viscous organogels in the presence of suitable organic solvents and also water-induced organogels upon addition of water to the organogel. These mixtures show shear-thinning properties and are birefringent. The behavior and properties of the organogels have been studied through rheology, DSC, and SAXS experiments. The organogels possess the same antioxidant properties of the original L-ascorbic acid ring and can be used to solubilize and protect valuable organic molecules.
\end{abstract}

\section{Introduction}

Vitamin $\mathrm{C}$ is a powerful, water soluble antioxidant. It inhibits free radical induced damages that are probably involved in a variety of health problems. ${ }^{1,2}$ It is active especially against lipid peroxidation promoted by active oxygen species (AOS). ${ }^{3}$ Because of its large reducing power $\left(E^{\circ \prime}=+0.23 \mathrm{~V}\right)$ it can be used to protect more valuable materials that are sensitive to radical attack, such as carotenoids and tocopherols. ${ }^{4}$ However, since ascorbic acid is poorly soluble in organic solvents, its applications are restricted to aqueous environments. In order to expand its reducing properties to hydrophobic media, several amphiphilic vitamin $\mathrm{C}$ derivatives have been synthesized and studied. ${ }^{3-13}$

In previous papers, we reported the synthesis and physicochemical characterization of water dispersions of single chain 6- $O$-ascorbyl alkanoates (ASC $n, n$ being the number of atoms in the alkanoate chain). ${ }^{3-12}$ In these molecules the primary hydroxyl group of the ascorbic acid ring in position 6 is linked to a hydrophobic side chain, while the other $-\mathrm{OH}$ residues are still available for hydrogen bonding and solvation. The efficient and strong redox activity of vitamin $C$ is still retained. ${ }^{3,4}$ The single chain derivatives, once hydrated, produce lamellar phases (coagels), micelles, or gel-like dispersions, depending on the chain length $(8 \leq n \leq 16)$ and temperature. . $^{8,10}$

More recently, we have described the preparation and selfassembly of a vitamin C-based bolaamphiphile (BOLA12), with two ascorbic acid moieties as polar headgroups, linked by a dodecanedioic acid moiety. ${ }^{14}$ BOLA12 forms monodisperse hollow nanotubes in water that turn into micellar dispersions upon heating. Because of their peculiar phase behavior and their redox functionalities, these nanoassemblies represent an excellent prospect for the delivery of hydrophobic and sensitive drugs. ${ }^{12-14}$

\footnotetext{
* Corresponding author. Fax: +39 (055) 457-3036. E-mail: pln@ csgi.unifi.it. Internet: http://www.csgi.unifi.it/.

University of Florence

$\doteqdot$ Australian National University.
}

Organogels are one of the most interesting and promising examples of self-assembled soft matter. They can be produced by low-molecular-mass organic gelators (LMOGs) and organic solvents. The addition of tiny amounts of water usually results in a different appearance, viscosity, and stability. ${ }^{15}$ Noncovalent interactions, i.e., hydrogen bonding, $\pi-\pi$ stacking, van der Waals, and electrostatic interactions, are involved and balance in the formation and stabilization of physical gels, while polymer gels are kept together by covalent bonds. ${ }^{16,17}$ The self-assembly of the organogelator monomers in a proper solvent leads to the formation of long fibrils that entangle and form a 3D tightly packed network that entraps enormous amounts of solvent even when the concentration of the organogelator is very small, typically below $2 \%$. Organogels are temporally stable and thermoreversible. Above a characteristic temperature they turn into free-flowing sols and revert to the original state upon cooling. ${ }^{17}$ Their applications are numerous and growing in number. ${ }^{15-17}$ One of the features most investigated of these nanomaterials is the structural properties that an organogelator must possess in order to produce an organogel. In order to be a good candidate for the formation of organogels, an LMOG must fulfill some structural and physical requirements, such as quite a high bulk melting point, a low solubility in the liquid (so as to produce a microphase separation), some anisotropy on the molecular level, and it must carry suitable groups in order to establish hydrogen bonds. ${ }^{17}$

With these premises, vitamin $\mathrm{C}$ amphiphilic derivatives seem to possess the main requisites to produce organogels. In fact, the mp of ascorbyl esters is quite high, ${ }^{9}$ their solubility in organic solvents is moderate (actually they are purified through recrystallization from $\mathrm{Et}_{2} \mathrm{O}$ /petroleum ether or washed with cold hydrocarbons), the anisotropy is guaranteed by the rigid fivemembered ring and their chirality, the ascorbic ring is separated from the hydrophobic tail by a flexible $-\mathrm{CHOH}-\mathrm{CH}_{2}-\mathrm{O}-$ $\mathrm{CO}-$ spacer, and the headgroup contains several polar residues that are able to establish hydrogen bonds with vicinal headgroups and with proper polar solvents. 

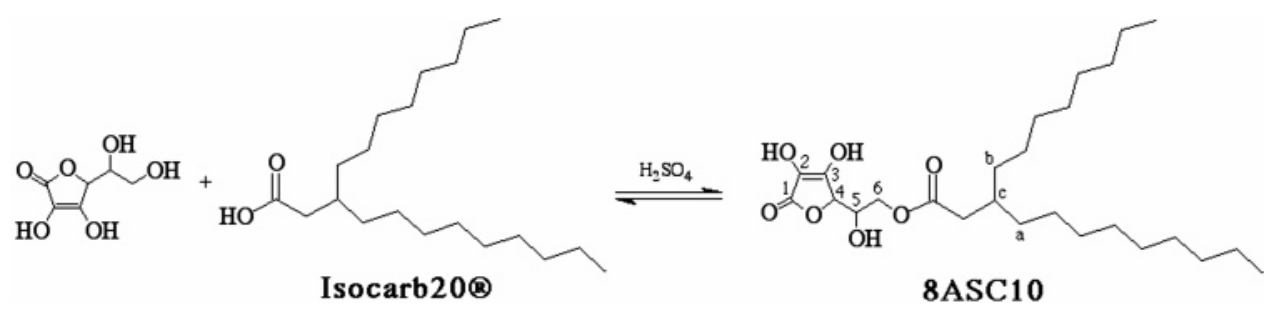

Figure 1. Synthesis and structure of $8 \mathrm{ASC} 10$.

We have already reported an extensive investigation on the formation and properties of the assembled nanomaterials that are formed in water by single chain ascorbyl alkanoates (ASC $n$ ) or by a $\mathrm{C}_{12}$-bolaamphiphile that carries two ascorbic acid units as polar headgroups. The properties of such dispersions depend on the nature of the surfactant, on its concentration, and on temperature. ${ }^{8}$ Moreover, in the case of ASCn we also studied the effect of electrolytes ${ }^{10}$ and neutral cosolutes ${ }^{9,11}$ on the solgel or coagel-gel phase transitions, as well as the effect of $\mathrm{pH} .{ }^{10}$ The experiments confirmed that the polar headgroups dominate in determining the morphology of the nanoassemblies.

To increase the hydrophobicity of vitamin $\mathrm{C}$ derivatives, and concomitantly to change curvature at the oil/surfactant/water interface from normal to reverse, we synthesized a new double chain surfactant, the 2-octyl-dodecanoyl-6-O-ascorbic acid (shortly 8ASC10). Its solid-state properties were studied via X-ray diffraction (XRD), small-angle X-ray-scattering (SAXS), and differential scanning calorimetry (DSC). This compound forms stable organogels in the presence of organic solvents, with or without water. The nanostructured assemblies keep the same antioxidant properties of vitamin C. Rheology, DSC, XRD, and SAXS experiments were also carried out to characterize these organogels. This is the first report of the formation of organogels from a vitamin C-based organogelator. As vitamin C-based LMOGs keep the same antioxidant power of the parent molecule, the resulting organogels are expected to possess this important functionality that extends their application and uses to the solubilization, storage, and protection of valuable but oxidizable materials.

\section{Materials and Methods}

Isocarb20 was a kind gift from Sasol GmbH (Germany). All reagents (analytical grade) were purchased from Fluka (Milan, Italy) and used without further purification. Isocarb20 and L-ascorbic acid were dried in a desiccator under vacuum and over $\mathrm{P}_{2} \mathrm{O}_{5}$ for $24 \mathrm{~h}$ before use. All the other chemicals were used as received. NMR spectra were recorded with a Varian Jemini 200 spectrometer. Fourier Transform IR (FTIR) spectra were obtained with a BioRad FTS-40-PL spectrometer, using $\mathrm{KBr}$ pellets.

Synthesis. 8ASC10 was synthesized according to the procedure already reported in the literature, which involves the reaction in concentrated sulfuric acid between a carboxylic acid and the primary $-\mathrm{OH}$ group in position 6 of the vitamin $\mathrm{C}$ molecule. 5

An amount of $250 \mathrm{~mL}$ of concentrated sulfuric acid was placed in a two-neck round-bottom flask, with a gentle stream of bubbling argon for 30 min under magnetic stirring at room temperature to remove dissolved oxygen. Then, $25.0 \mathrm{~g} \mathrm{(142}$ mmol) of dry L-ascorbic acid was added and completely dissolved, followed by $15.0 \mathrm{~g}(48 \mathrm{mmol})$ of dry Isocarb20. The flask was equipped with a $\mathrm{CaCl}_{2}$ tube to prevent adsorption of water from the atmosphere and with a water bath at $37^{\circ} \mathrm{C}$. After $15 \mathrm{~h}$, the mixture was poured into a $2.5 \mathrm{~L}$ beaker filled with ice, and stirred until it reached room temperature. The solution was then transferred into a separatory funnel and treated with diethyl ether several times. The organic phase was collected and washed with distilled water, before being treated with sodium sulfate for $1 \mathrm{~h}$ and filtered. After evaporation of the solvent, a yellow film was obtained. The crude product was dissolved in methanol under reflux, and purified with charcoal. The purification was repeated twice. Finally, the material was washed with cold $i$-octane to remove the hydrophobic impurities (unreacted Isocarb20), and a white powder was obtained, which was kept in a desiccator over $\mathrm{P}_{2} \mathrm{O}_{5}$ and under vacuum. The purity of the final product was assessed by TLC (eluent $\mathrm{CHCl}_{3} / \mathrm{MeOH}$ 95:5 v/v) which showed the presence of a single spot at $R_{\mathrm{f}} \approx$ 0.30. Elemental analysis was made after freeze drying: C, calcd $66.35 \%$, exptl $66.64 \%$; $\mathrm{H}$, calcd $9.85 \%$, exptl $10.13 \%$. The addition of a few drops of $\mathrm{Br}_{2} / \mathrm{CH}_{2} \mathrm{Cl}_{2}$ to a solution of $8 \mathrm{ASC} 10$ in acetone brings about instantaneous discoloration of bromine, showing that the vitamin $\mathrm{C}$ double bond is still active in 8ASC10. IR ( $\mathrm{KBr}$ disc) $\bar{v} / \mathrm{cm}^{-1} 3586-3100(\mathrm{O}-\mathrm{H}), 2927,2850$ $(\mathrm{C}-\mathrm{H}), 1762,1716(\mathrm{C}=\mathrm{O}), 1670(\mathrm{C}=\mathrm{C}), 1471,1340\left(\mathrm{CH}_{2}\right)$, $1180(\mathrm{C}-\mathrm{O}), 743\left(\mathrm{CH}_{2}\right)$. NMR $(\delta \mathrm{H}, 300 \mathrm{MHz}, \mathrm{ppm}$; in DMSO; see Figure 1 for numbering) for 8ASC10: 0.886 (triplet, $\mathrm{CH}_{3}$, $J=6.3 \mathrm{~Hz}$ ), 1.257 (multiplet, $14 \times \mathrm{CH}_{2}$ ), 1.479 (multiplet, $\mathrm{CH}_{2} \mathrm{a}$ and b), 2.35 ( $\mathrm{CH} \mathrm{c}$ ), 2.54 (DMSO), $3.374\left(\mathrm{H}_{2} \mathrm{O}\right), 3.985$ and 4.083 (multiplet, 6- $\mathrm{CH}_{2}$ and 5-CH), 4.664 (duplet, 4- $\mathrm{CH}, J$ $=1.5 \mathrm{~Hz}$ ), 5.367 (singlet, 5-OH), 8.471 (singlet, 3-OH), 11.202 (singlet, 2-OH). For Isocarb20: 0.890 (triplet, $2 \times \mathrm{CH}_{3}, J=$ $6.6 \mathrm{~Hz}$ ), between 1.268 and 1.600 (multiplet, $16 \times \mathrm{CH}_{2}$ ), 2.208 $\left(9-\mathrm{CH}, J_{1,21}=8.1 \mathrm{~Hz}, J_{1,11}=5.4 \mathrm{~Hz}\right), 2.54$ (DMSO), 3.381 $\left(\mathrm{H}_{2} \mathrm{O}\right), 12.059$ (singlet, $\mathrm{OH}$ ).

Sample Preparation. The 8ASC10 powder was freeze-dried overnight. Vesicle preparation was performed following the thinfilm hydration procedure. In a round-bottomed flask the proper amounts of $8 \mathrm{ASC} 10(1 \% \mathrm{w} / \mathrm{w})$ and of the cosurfactants (LDS and ASC10, both $0.5 \% \mathrm{w} / \mathrm{w}$ ) were mixed and dissolved with chloroform. The solvent was evaporated slowly, and then the flask was left overnight under vacuum. Water was added to the film; and the dispersion was heated to $70{ }^{\circ} \mathrm{C}$ and sonicated for $10 \mathrm{~min}$. This procedure leads to the formation of polydispersed multilamellar vesicles. In order to obtain unilamellar aggregates, the freeze-and-thaw method was used: the dispersion was frozen in liquid nitrogen and then heated to $70{ }^{\circ} \mathrm{C}$. The cycle was repeated at least six times. Finally high-pressure extrusion through $0.1 \mu \mathrm{m}$ pores at $70{ }^{\circ} \mathrm{C}$ produced a homogeneous and quite monodisperse solution of vesicles that were examined by QELS.

X-ray Diffraction (XRD). XRD diffractograms were obtained with a powder Bruker D8 Advance Diffractometer (BRUKER axs), using Bragg-Brentano geometry. $\lambda=1.5405$ $\AA(\mathrm{Cu} \mathrm{K} \alpha)$. Experiments were carried out in the range $1.0^{\circ} \leq$ $2 \theta \leq 40^{\circ}$, with a step size of $0.01^{\circ}$, a time/step of $5 \mathrm{~s}$, setting voltage and current at $30 \mathrm{kV}$ and $20 \mathrm{~mA}$, respectively.

Small-Angle X-ray-Scattering (SAXS). SAXS measurements were carried out with a HECUS SWAX-camera (Kratky) 
equipped with a position-sensitive detector (OED 50M) containing 1024 channels of width $54 \mu \mathrm{m} . \mathrm{Cu} \mathrm{K}_{\alpha}$ radiation of wavelength, $\lambda=1.542 \AA$, was provided by a Seifert ID-3003 $\mathrm{X}$-ray generator (sealed-tube type), operating at a maximum power of $2 \mathrm{~kW}$. A $10 \mu \mathrm{m}$ thick nickel filter was used to remove the $\mathrm{Cu} \mathrm{K}_{\beta}$ radiation. The sample-to-detector distance was 273 $\mathrm{mm}$. The volume between the sample and the detector was kept under vacuum during the measurements to minimize scattering from the air. The Kratky camera was calibrated using silver behenate, which is known to have a well-defined lamellar structure $(d=58.48 \AA) .{ }^{18}$ Scattering curves were obtained in the Q-range between 0.007 and $0.54 \AA^{-1}$, Q being the scattering vector $(4 \pi \sin \theta) / \lambda$, and $\theta$ the scattering angle. The gel samples were filled into a $1 \mathrm{~mm}$ quartz capillary and centrifuged for about $5 \mathrm{~min}$ at $1000 \mathrm{rpm}$. Birefringence was checked after centrifugation in order to confirm the organogel phase. The temperature was controlled by a Peltier element, with an accuracy of $\pm 0.1{ }^{\circ} \mathrm{C}$. A temperature-scan between 20 and 50 ${ }^{\circ} \mathrm{C}$ was performed by using a temperature-controller (KPR) with the MTC-program (HECUS). No solvent evaporation was found after each temperature scan. All scattering curves were corrected for the solvent and the empty cell contribution considering the relative transmission factor. SAXS curves were iteratively desmeared using the procedure reported by Lake. ${ }^{19}$

Scattering Length Densities (SLDs). SLDs $(\rho)$ for the polar headgroups, the hydrophobic tails, and the solvent ( $i$-octane) were calculated as

$$
\rho=\sum_{\mathrm{i}} b_{\mathrm{i}} \frac{d N_{\mathrm{A}}}{M_{\mathrm{W}}}
$$

where $i$ runs over the atoms composing the scattering object, $b_{\mathrm{i}}$ is the X-ray scattering cross-section for atom i, $d$ is the bulk density of the object, $M_{\mathrm{W}}$ its molecular weight, and $N_{\mathrm{A}}$ the Avogadro number. In order to calculate the density, we considered a hydrophobic tail volume of $460 \AA^{3}$ and a polar head volume of $367 \AA^{3}$. Both values were obtained with molecular mechanics calculations averaging on a micelle composed of 22 monomers of 8ASC10 in vacuum, with a MM+ force field, ${ }^{20}$ and Block Diagonal Newton-Raphson as the minimization algorithm. We also included water in the calculation for the micelle core $\left(v_{\mathrm{w}}=30 \AA^{3}\right)$ assuming that all the water added to the sample $(1 \% \mathrm{w} / \mathrm{w})$ is confined in the hydrophilic pool of the inverted micelle. Therefore we obtained $\rho_{\text {i-octane }}=6.78 \times 10^{-6} \AA^{-2}, \rho_{\text {tails }}=8.43 \times 10^{-6} \AA^{-2}$, and $\rho_{\text {head }}$ $=8.55 \times 10^{-6} \AA^{-2}$. The tails SLD was just taken as an initial value, for it was allowed to change freely during the fitting with the core-shell models to include the effect of solvent insertion between the surfactant tails, that obviously lowers the contrast. In fact, we noticed that the $i$-octane contrast lowered to 0 during the fitting procedure and decided to adopt a uniform model approach, as described below.

Uniform Sphere Model. The form factor used for monodisperse spherical particles assuming a uniform SLD is given by ${ }^{21}$

$$
P(\mathrm{Q})=\frac{k}{V_{\mathrm{p}}}\left[\frac{3 V_{\mathrm{p}} \Delta \rho(\sin (\mathrm{Q} R)-\mathrm{Q} R \cos (\mathrm{Q} R))}{(\mathrm{Q} R)^{3}}\right]^{2}+b
$$

where $k$ is a scale factor, $V_{\mathrm{p}}$ is the particle volume, $\Delta \rho=\left(\rho_{\text {head }}\right.$ $\left.-\rho_{i-\text { octane }}\right)$ is the scattering contrast, $R$ is the sphere radius, and $b$ is the additive background.

Uniform Cylinder Model. The form factor used for monodisperse cylindrical particles assuming a uniform SLD is calculated as $^{22}$

$$
\begin{aligned}
& P(\mathrm{Q})=\frac{k}{V_{\mathrm{p}}} \int_{0}^{\pi / 2} \\
& {\left[2 \Delta \rho V_{\mathrm{p}} \frac{\sin (\mathrm{Q} H \cos \alpha)}{\mathrm{Q} H \cos \alpha} \frac{\sin (\mathrm{Q} R \sin \alpha)-\mathrm{Q} R \sin \alpha \cos (\mathrm{Q} R \sin \alpha)}{(\mathrm{Q} R \sin \alpha)^{3}}\right]^{2} \sin \alpha \mathrm{d} \alpha+b}
\end{aligned}
$$

where $H$ is half the cylinder's length, $\alpha$ is the angle between the scattering vector $\mathrm{Q}$ and the cylinder axis, and $b$ is the additive background. The integral over $\alpha$ averages the form factor over all possible orientations of the cylinder with respect to Q.

Differential Scanning Calorimetry (DSC). DSC experiments were carried out with a Q1000 TA Instruments apparatus, using airtight aluminum pans, sealed under nitrogen atmosphere. The heating rate was $0.5^{\circ} \mathrm{C} / \mathrm{min}$, unless otherwise specified. The phase transition temperatures were taken as the temperatures of the endothermic peaks, and the enthalpic changes were determined by integrating the heat flow curves. ${ }^{10}$

Reducing Activity. The reducing activity (RA, \%) was evaluated by measuring the absorbance at $517 \mathrm{~nm}$ of a DPPH $(\alpha, \alpha$-diphenyl- $\beta$-picrylhydrazyl) solution in ethanol $(0.1 \mathrm{mM})$ before $\left(A_{0}\right)$ and after $\left(A_{20}\right) 20 \mathrm{~min}$ from the addition of an equal volume of the sample $(0.1 \mathrm{mM}$ in ethanol or chloroform $)$, as $\operatorname{RA}(\%)=100\left(A_{0}-A_{20}\right) / A_{0} .^{3}$

Organogels. 8ASC10 was dissolved in the organic solvent at a concentration ranging between 1 and $5 \% \mathrm{w} / \mathrm{w}$ using a vortexer and heating the sample. For the water-induced organogels, water was added and the mixture was shaken again with a vortexer. A milky physical gel was then formed. Repeated heating-cooling cycles were carried out to homogenize and equilibrate the gel, that appears as a colorless very viscous liquid.

Phase Behavior. The gel samples were prepared by weighing the appropriate amounts of 8ASC10, water, and hydrocarbon (cyclohexane or $i$-octane) directly in the vials. The vials were equipped with a small magnetic anchor, tightly closed, and thermostatted in a water bath. The temperature was increased stepwise by $1{ }^{\circ} \mathrm{C}$. The sol-gel transition point was taken as the temperature at which the gel became liquid.

Rheology. The rheological behavior of the organogels was studied with a Paar Physica UDS-200 rheometer with a parallelplate geometry. The temperature of the sample was controlled with a Peltier element. A plate-plate geometry was used (20 $\mathrm{mm}$ diameter, $0.3 \mathrm{~mm}$ gap) with serrated surfaces so as to prevent sliding due to the liquid film expelled by some dilute samples. The frequency sweep measurements were performed from $0.001-15 \mathrm{~Hz}$. The rheological behavior was determined by measuring the complex viscosity and the $G^{\prime}$ and $G^{\prime \prime}$ moduli in an oscillation regime (the amplitude of the oscillation was $0.5 \%)$ in the linear viscoelastic region of deformations. The temperature was $20.00 \pm 0.01{ }^{\circ} \mathrm{C}$. The dependence of the rheological parameters $G^{\prime}, G^{\prime \prime}$, and $\eta^{*}$ from the oscillation frequency are obtained from the phase lag between the applied shear stress and the related flow and from the ratio between the amplitudes of the imposed oscillation and the response of the gel. $\eta^{*}$ is obtained directly from $G^{\prime}$ and $G^{\prime \prime}$ as

$$
\left|\eta^{*}(\omega)\right|=\sqrt{\frac{G^{\prime 2}(\omega)+G^{\prime 2}(\omega)}{\omega^{2}}}
$$

where $\omega$ is the angular frequency $(\omega=2 \pi v)$.

Light Scattering. Quasi elastic light scattering (QELS) experiments were carried out with a Brookhaven Instruments apparatus, consisting of a goniometer BI200SM, a digital correlator BI9000AT, and a photomultiplier ENI 9863B/350. 


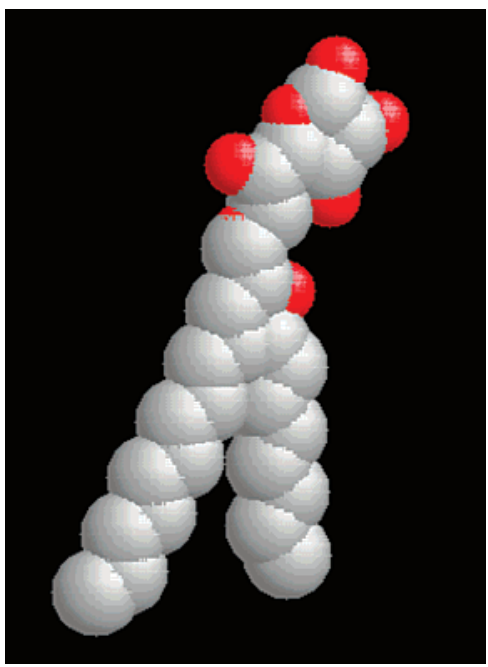

Figure 2. CPK spacefilling model of 8ASC10. Carbons and oxygens are gray and red, respectively (hydrogens have been hidden for clarity).

A vertically polarized light emitted by a diode-laser Nd:YAG $(\lambda=532 \mathrm{~nm})$ was used. The refractive index matching liquid was decahydronaphthalene, and the measurements were performed at a scattering angle of $90^{\circ}$, at $25^{\circ} \mathrm{C}$.

\section{Results and Discussion}

2-octyl-dodecanoyl-6- $O$-ascorbic acid (8ASC10, see Figure $1)$ is a white, sticky powder. It was synthesized from 2-octyldodecanoic acid (Isocarb20) and L-ascorbic acid. 8ASC10 carries a hydrophilic moiety (the L-ascorbic acid and the ester residue) bound to a hydrophobic tail. It is therefore expected to act as a surfactant. 8ASC10 is moderately soluble in organic solvents but almost insoluble in water at room temperature. In the presence of liquid alkanes (i.e., hexane, cyclohexane, octane, decane) it forms viscous organogels. Figure 2 shows the minimized CPK (Corey-Pauling-Koltun) model of 8ASC10. According to Tanford's rule, ${ }^{22}$ the length of the hydrophobic chain in the fully stretched conformation is $11.5 \AA\left(L_{\mathrm{h}}\right)$, and the volume of the entire hydrophobic region is $512 \AA^{3}\left(v_{\mathrm{h}}\right)$.

8ASC10 has the same characteristics of typical double chained surfactants, e.g., dimethyldidodecyl ammonium bromide (DDAB): weak oil solubility, chain length, oil uptake, and Krafft temperature, that make them such ideal microemulsion promoters. ${ }^{23,24}$ Such surfactants form three-component stable phases at very low water content (about 1\%). However, unlike the quaternary ammonium cationic headgroup, the L-ascorbic acid moiety in 8ASC10 possesses several hydrophilic residues that organize in hydration interactions, and consequently rearrange, in a way that recalls the sol-gel phase behavior of $i$-octane $/ \mathrm{Cu}(\mathrm{AOT})_{2} /$ water mixtures. $^{25-27}$

Solid Phase. The pure 8ASC10 shows a very large reducing activity above $95 \%$ (evaluated with the DPPH ( $\alpha, \alpha$-diphenyl$\beta$-picrylhydrazyl) method), comparable to that of L-ascorbic acid and its derivatives. ${ }^{3}$ DSC experiments on the solid material indicated a mp of $66^{\circ} \mathrm{C}$ and a $\Delta H_{\text {melt }}$ of $35.7 \mathrm{~kJ} / \mathrm{mol}$. The parent double chain carboxylic acid (Isocarb20) has a mp of $37.2^{\circ} \mathrm{C}$ and a $\Delta H_{\text {melt }}$ of $42.4 \mathrm{~kJ} / \mathrm{mol}$. The XRD profile of the pure solid (see Figure 3 ) revealed the presence of crystallinity, with the most intense peak corresponding to a spacing of $18 \AA$, which is about the calculated length of the molecule. The peaks between $18^{\circ}$ and $25^{\circ}$ are due to the compact packing of the hydrophobic tails. ${ }^{11,14}$ Increasing the temperature to 30,50 , and

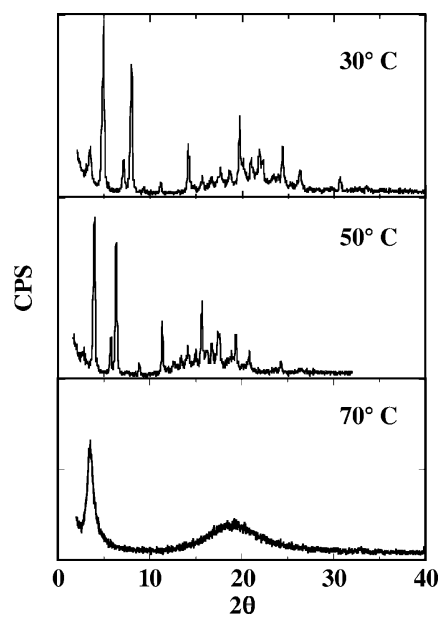

Figure 3. XRD profiles for solid $8 \mathrm{ASC} 10$ as a function of temperature.

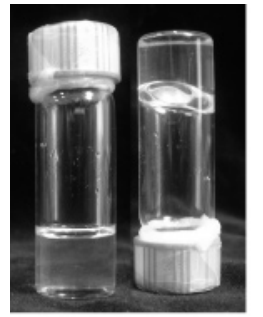

(a)

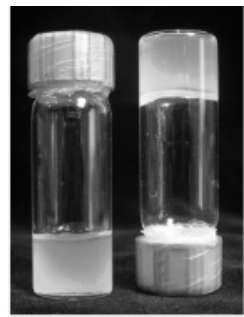

(b)

Figure 4. Organogels obtained from (a) 8ASC10 and cyclohexane $(5: 95 \mathrm{w} / \mathrm{w})$; (b) $8 \mathrm{ASC} 10$, water, and cyclohexane $(5: 1: 94 \mathrm{w} / \mathrm{w} / \mathrm{w})$.

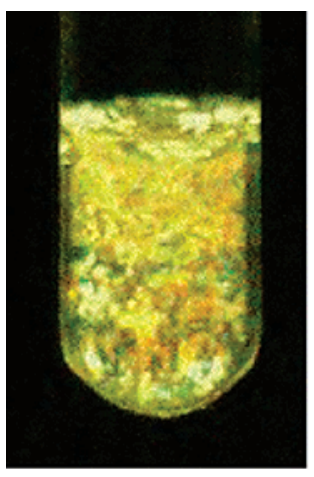

(a)

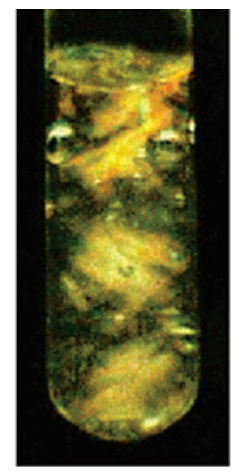

(b)

Figure 5. Birefringence shown by an organogel obtained from (a) 8 ASC10 and $i$-octane $(5: 95 \mathrm{w} / \mathrm{w})$; (b) 8 ASC10, water, and $i$-octane $(5: 1: 94 \mathrm{w} / \mathrm{w} / \mathrm{w})$.

$70{ }^{\circ} \mathrm{C}$ results in the appearance of a new peak at $3.5^{\circ}(25 \AA)$, with the simultaneous loss of crystallinity as the sample is heated up.

The peak centered at $25 \AA$ was revealed also by SAXS experiments, but disappeared in the freeze-dried solid, suggesting that it is due to a hydrated structure. The same effect of hydration on the XRD profiles of the pure powder was found in the single chain vitamin C-based surfactants (ASCn). ${ }^{11}$

Aqueous Dispersions. 8ASC10 is poorly soluble in water, but in the presence of cosurfactants such as phospholipids, lithium dodecylsulfate (LDS), and 6-O-ascorbyl-decanoate (ASC10), it forms vesicles by extrusion. Dynamic light scattering measurements indicated the presence of curved bilayers with an average diameter of $90 \mathrm{~nm}$ (polydispersity 21\%) from mixtures containing $1 \%$ of $8 \mathrm{ASC} 10,0.5 \%$ of $\mathrm{LDS}$, and $0.5 \%$ of ASC10.

Organogels. 8ASC10 produces organogels when dispersed in the proper organic solvent. A small amount of water can be incorporated in the gel. We studied in detail the dispersions of 

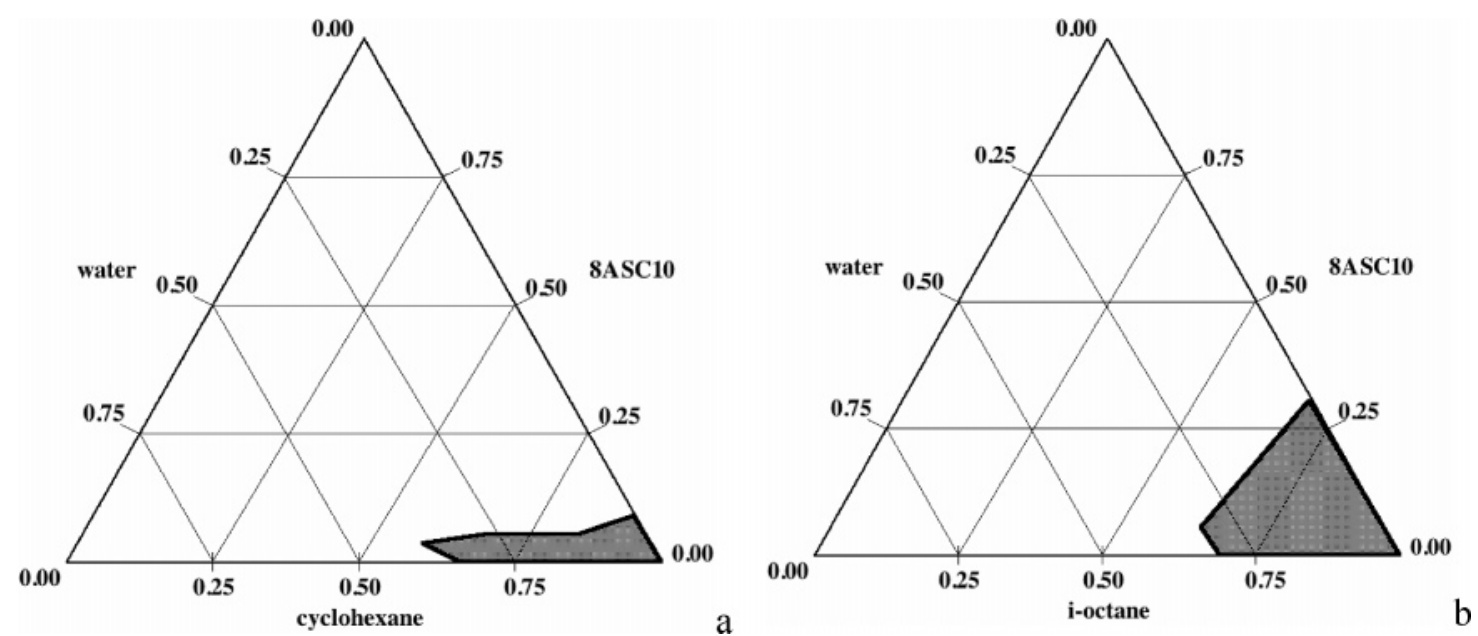

Figure 6. Ternary phase diagrams for $8 \mathrm{ASC} 10 /$ water/cyclohexane (a) and $8 \mathrm{ASC} 10 /$ water/i-octane (b) mixtures at $20^{\circ} \mathrm{C}$.

8ASC10 in cyclohexane or $i$-octane, with and without water. The water-free organogels are transparent and highly viscous and show shear-thinning properties (Figure 4a). The waterinduced organogels with the same oil phase are mechanically stronger than the water-free samples (Figure 4b); they are not transparent and may show shear-thinning properties depending on the water content. All organogels are monophasic birefringent systems at room temperature as shown in Figure 5, indicating the presence of domains with a compact ordered orientation of the aggregates in the samples.

The organogels were prepared with different amounts of water, ranging between 0 and $40 \%$. When the water content is large, however, the gel is not thermodynamically stable and the phase separates after some days. Their stability is increased by higher amounts of surfactant. The ternary phase diagrams of 8ASC10/water/cyclohexane and 8ASC10/water/i-octane systems at $20{ }^{\circ} \mathrm{C}$ are shown in Figure 6 .

The samples exhibit a sol-gel phase transition, which is typical for physical gels, where the 3D network is held together by hydrogen bonding and van der Waals interactions. ${ }^{16,28,29}$ Because of their high viscosities, repeated heating-cooling cycles are necessary to obtain a homogeneous and equilibrated organogel. Samples with large water contents exhibit a phase separation about $5{ }^{\circ} \mathrm{C}$ below the sol-gel transition. When the surfactant concentration is about $1 \%$, some water is necessary to build up the gel. When the amount of 8ASC10 is increased to $5 \%$, the gels are more stable against phase separation, and the sol-gel temperature decreases. The effect of water on the stability of these gels is similar to that already observed for lecithins, where long cylindrical inverted micelles are stabilized by the addition of water that remains confined in the polar headgroup regions. ${ }^{30,31}$ In those systems, water forms bridges between the phosphate groups through hydrogen bonding. In the case of 8ASC10, the presence of several hydrated polar groups in the ascorbic acid moiety, and particularly of the acidic hydroxyl residue in position 3, leads to the formation of hydrogen bonds between the surfactant's headgroups that stabilize the self-assembled structure. Moreover, since the chain length of the oil molecules does not exceed that of the surfactant chains, the oil phase penetrates into the double chained surfactant region. In this way the curvature at the interface sets the structure of the aggregate. ${ }^{24}$

Figure 7 shows the DSC runs for the organogels produced by $8 \mathrm{ASC} 10$ with cyclohexane with and without water. The $1 \%$ water-induced organogel (-) shows an endothermic peak at about $32{ }^{\circ} \mathrm{C}$, that corresponds to the sol-gel transition tem-

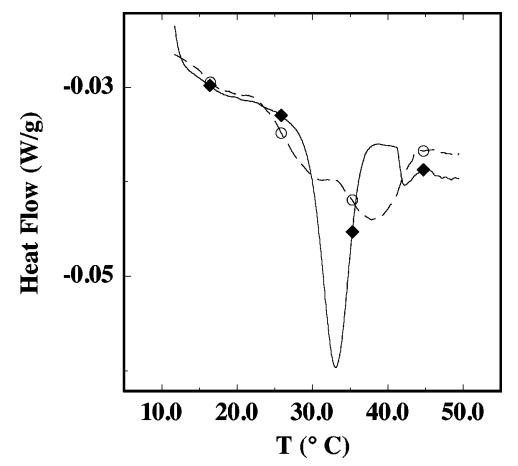

Figure 7. DSC profile for organogel samples. $\bullet$, 8ASC10/water/ cyclohexane $(5: 1: 94 \mathrm{w} / \mathrm{w} / \mathrm{w}) ;$ O, 8ASC10/water/cyclohexane $(5: 0: 95$ $\mathrm{w} / \mathrm{w} / \mathrm{w})$.

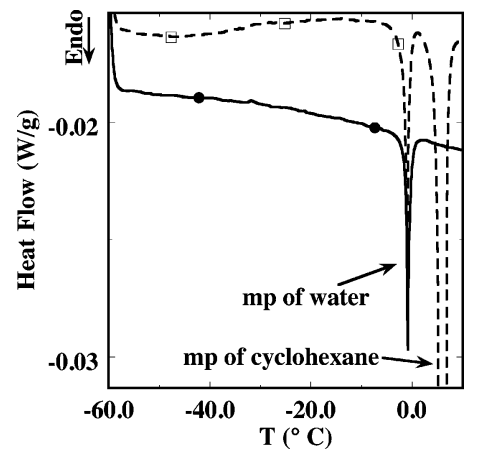

Figure 8. DSC thermograms for the evaluation of the amount of strongly bound water in a sample of $8 \mathrm{ASC} 10 /$ water/i-octane $(\mathbf{0})$; and of 8ASC10/water/cyclohexane ( $\square$ ). In both cases the organogel composition is $5: 1: 94 \mathrm{w} / \mathrm{w} / \mathrm{w}$.

perature. The enthalpy change for the sol-gel transition is quite small $\left(3.8 \mathrm{~J} / \mathrm{g}_{\text {sample }}\right)$ and reflects the weak interactions between the components. The water-free organogel $(---)$ shows a broader peak shifted at a slightly higher temperature.

DSC experiments can be used to evaluate the amount of strongly bound water molecules. ${ }^{11,14}$ In fact, hydration water is essentially "frozen" and remains strongly bound to the surfactant headgroups while the sample is heated (see Figure 8). Samples were first cooled down quickly to $-60{ }^{\circ} \mathrm{C}$ in order to avoid separation of ice from the dispersion. They were then heated up to $10^{\circ} \mathrm{C}$. The amount of strongly bound water over the whole water content, $W_{\mathrm{b}}(\%)$, can be obtained from the decrease of the area of the endothermic peak associated with the melting of bulk water according to eq 5 . The corresponding number of 


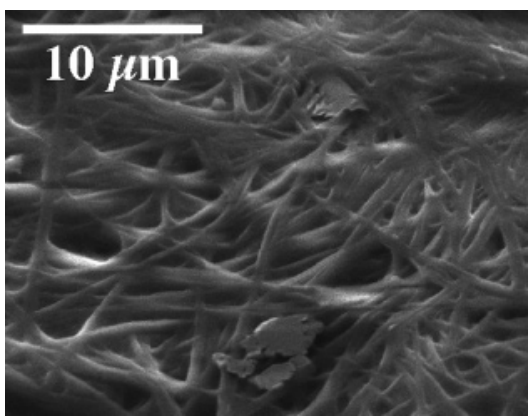

Figure 9. SEM micrograph showing the entangled network produced in an organogel obtained from 8ASC10/water/cyclohexane after freeze drying.

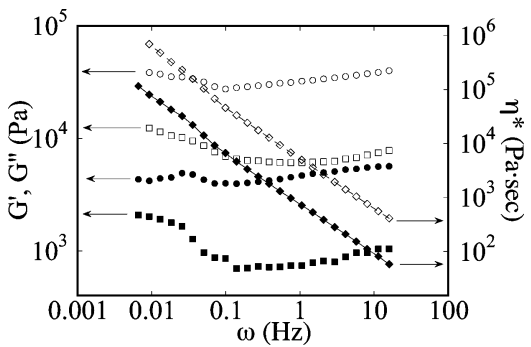

Figure 10. Rheology experiment performed on organogels made up of $8 \mathrm{ASC} 10 /$ cyclohexane $(5: 95 \mathrm{w} / \mathrm{w})$ (filled symbols) and of $8 \mathrm{ASC} 10 /$ water/cyclohexane $(5: 1: 94 \mathrm{w} / \mathrm{w} / \mathrm{w})$ (open symbols). The storage modulus $G^{\prime}$ (circles) and the loss component $G^{\prime \prime}$ (squares) are plotted on the left $y$-axis, the complex modulus $\eta^{*}$ is represented on the right $y$-axis $(\diamond$ and $\diamond)$.

strongly bound water molecules per polar headgroup, $N_{\mathrm{b}}$, is then calculated from $W_{\mathrm{b}}(\%)$ from eq $6:^{11}$

$$
\begin{gathered}
W_{\mathrm{b}}(\%)=100\left(1-\frac{\Delta H_{\mathrm{exp}}}{333.4 x_{\mathrm{w}}}\right) \\
N_{\mathrm{b}}=\frac{M_{\mathrm{s}} W_{\mathrm{b}}}{M_{\mathrm{w}} P_{\mathrm{s}}}
\end{gathered}
$$

where $333.4 \mathrm{~J} / \mathrm{g}$ is the enthalpy change of melting for pure water as reported in the literature,,$^{32} \Delta H_{\exp }$ (in $\mathrm{J} / \mathrm{g}_{\text {sample }}$ ) is the measured peak area, $x_{\mathrm{w}}$ is the weight fraction of water in the sample $(0.01)$, $P_{\mathrm{s}}$ is the weight fraction of the surfactant $(0.05), M_{\mathrm{s}}$ and $M_{\mathrm{w}}$ are the molecular masses of $8 \mathrm{ASC} 10$ and of water, respectively. DSC experiments run for 8ASC10/water/cyclohexane and 8ASC10/water/i-octane specimens provided values for $N_{\mathrm{b}}$ of about $3.0 \pm 0.2$ for both samples. In the case of the aqueous dispersions of the single chain ASC12 (40\%) and of the bolaform BOLA12 (3\%) surfactant, we found approximately seven molecules of water strongly bound to the ascorbic acid polar headgroups. ${ }^{11,14}$

SEM micrographs were taken on gel samples after freezedrying. Figure 9 shows that an entangled structure has formed, with several interconnecting and overlapped rodlike ribbons, with an average diameter of about $0.3-1 \mu \mathrm{m}$.

Dynamic mechanical measurements were performed to explore the viscoelastic properties of two gel phases. Figure 10 shows the storage modulus $\left(G^{\prime}\right)$ and the loss component $\left(G^{\prime \prime}\right)$ of the complex modulus $\left(\eta^{*}\right)$ as a function of frequency. The complex viscosity is given by eq 4 . The trends are those typical of a solidlike material: $G^{\prime}$ is always larger than the loss modulus in the observed region of frequencies without a crossover point of the shear moduli. This difference can be interepreted by recalling the rheological behavior of lecithin organogels. ${ }^{31}$ In

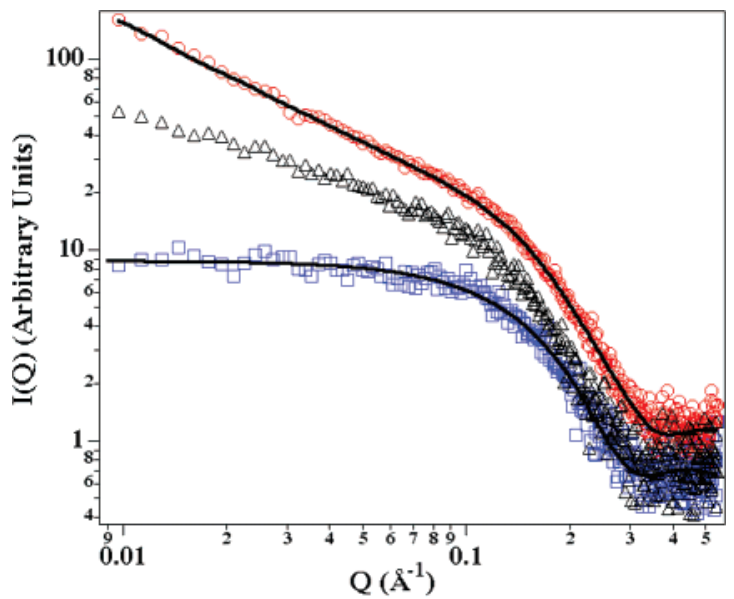

Figure 11. SAXS intensity distribution for an $8 \mathrm{ASC} 10 /$ water/i-octane $(5: 1: 94 \mathrm{w} / \mathrm{w} / \mathrm{w})$ organogel as a function of temperature: $20{ }^{\circ} \mathrm{C}$ (red circles), $30^{\circ} \mathrm{C}$ (black triangles), and $50{ }^{\circ} \mathrm{C}$ (blue squares). The lines represent the fittings for the cylinder model at $20^{\circ} \mathrm{C}$ and for the sphere model at $50{ }^{\circ} \mathrm{C}$.

that case, the rheological behavior was explained in terms of the reptation model. ${ }^{31}$ Instead, for $p$-octylbenzohydroxamic acid (OBHA)/dodecane organogels, Terech et al. report a different rheological behavior, that, according also to SANS and SAXS data, indicates the presence of rigid bilayered ribbons or fibrils, that fuse and produce thicker fibers. ${ }^{33} 8 \mathrm{ASC} 10 /$ alkane systems show a similar behavior to that of $\mathrm{OBHA} / \mathrm{C}_{12} \mathrm{H}_{26}$ gels, with a storage modulus that is always larger than the loss modulus and with almost constant moduli over the investigated $\omega$ range. The results also indicate that the physical junctions in the network behave as permanent cross-links with long lifetimes, typical of other self-assembled systems. ${ }^{34}$ The solidlike state is also confirmed by the fact that both $G^{\prime}$ and $G^{\prime \prime}$ are rather independent of the frequency within the range investigated. Upon addition of water, $G^{\prime}, G^{\prime \prime}$, and $\eta^{*}$ increase about 1 order of magnitude. This fact is probably related to the hydration of the surfactant headgroups and the formation of hydrogen bonds between the $\mathrm{C}=\mathrm{O}$ and $\mathrm{O}-\mathrm{H}$ groups in the ascorbate moieties.

SAXS experiments were performed on the gel samples as a function of temperature. Figure 11 shows the profiles obtained for $8 \mathrm{ASC} 10 /$ water $/ i$-octane $(5: 1: 94 \mathrm{w} / \mathrm{w} / \mathrm{w})$ at $20^{\circ}, 30^{\circ}$, and $50{ }^{\circ} \mathrm{C}$.

In the low $\mathrm{Q}$ region, the change in slope from -1 to 0 indicates the occurrence of a structural transition from cylinders to spheres upon heating from $20^{\circ} \mathrm{C}$ to $50^{\circ} \mathrm{C}$. In order to extract the structural parameters in the two limiting cases, we checked several fitting procedures for different models. In all models we assumed that the contrast difference was mainly due to the interface that separates the polar headgroups from the hydrophobic tails. This was confirmed by a core shell approach where the scattering length density (free parameter) of the tails resulted equal to that of $i$-octane (fixed parameter). The continuous lines reported in Figure 11 show the best fitting results. A uniform, monodisperse, cylindrical form factor was used to fit the data at $20{ }^{\circ} \mathrm{C}$ (red circles). The cylinder radius and length came out to be about $9.8 \pm 1 \AA$ and $400 \pm 50 \AA$, respectively. It is important to recall that the cylinder length is very close to the maximum dimension accessible with the used experimental setup; therefore, its value represents the lower limit for the real dimension.

At higher temperatures, the SAXS profile indicates that a structural transition from cylinders to spheres takes place between $30^{\circ} \mathrm{C}$ and $50{ }^{\circ} \mathrm{C}$, in agreement with the DSC results. 


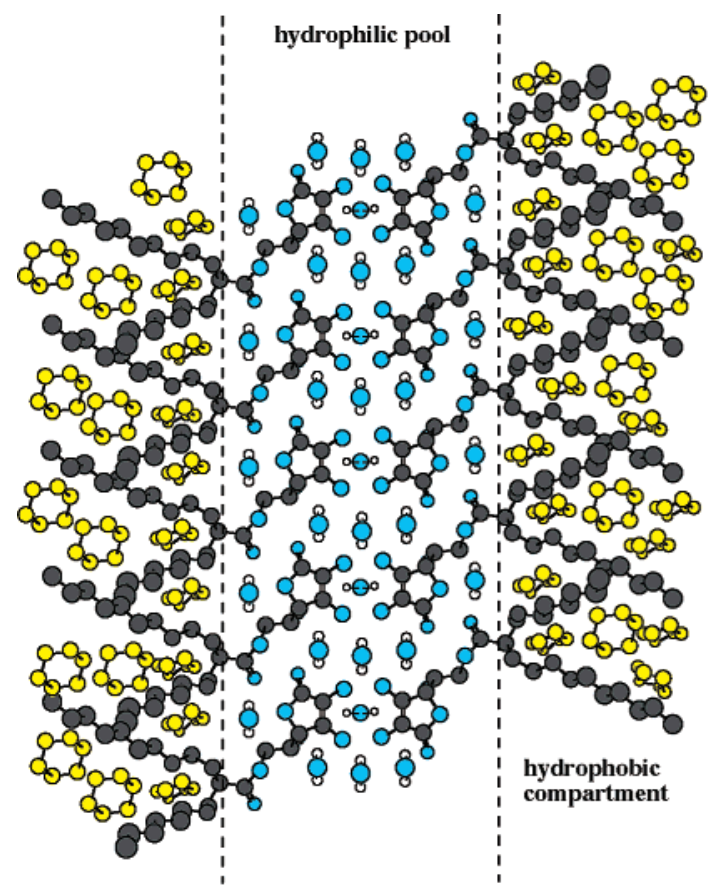

(a)

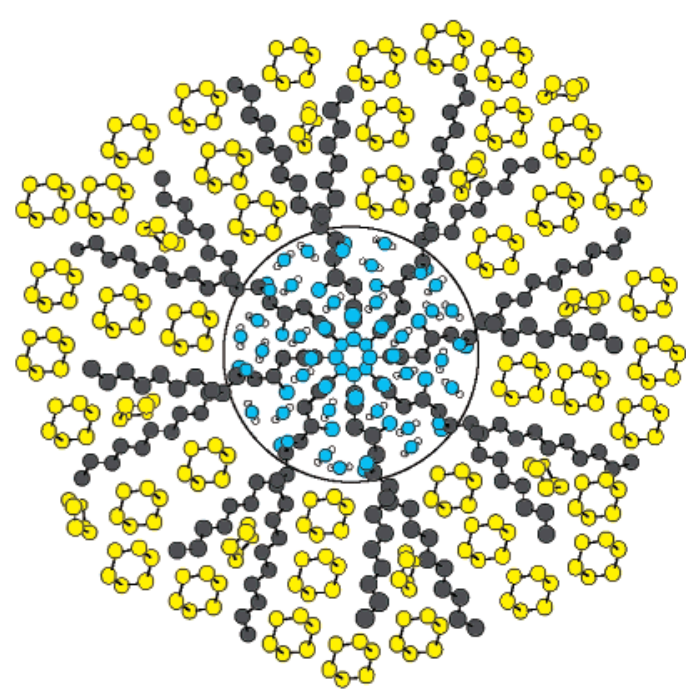

(b)

Figure 12. Side (a) and top (b) view of the cylinders in the organogels. The hydrophilic pool contains the vitamin $\mathrm{C}$ polar headgroups and water molecules. The hydrophobic compartment is filled with the 8ASC10 lipophilic chains and the solvent molecules.

The fitting of the $50{ }^{\circ} \mathrm{C}$ curve reveals the presence of inverted micelles with an average radius of about $13.3 \pm 1 \AA$.

The DSC and SAXS results seem to indicate the presence of long cylinders at $20^{\circ} \mathrm{C}$ in the $8 \mathrm{ASC} 10 /$ water/i-octane mixture. The dispersing phase contains the hydrocarbon (cyclohexane or $i$-octane), while water is confined in a narrow central core. The 8ASC10 surfactant acts as an emulsifier and self-assembles into an inverted structure, with the hydrophobic tails penetrating into the oil phase and the vitamin $\mathrm{C}$ head groups anchored in the hydrophilic pool. From molecular mechanics calculations, we determined the volume occupied by a single headgroup as $367 \AA^{3}$, and the length of the head group as $9.2 \AA$.

According to the DSC and SAXS results, the structure of the assembled nanostructures can be depicted as shown in Figure 12 (side and top view). The radius of the cylinder is $9.8 \AA$ and corresponds to the length of the headgroup (9.2 $\AA$ ). Water molecules occupy the available space in the hydrophilic pool and keep the ascorbic acid moieties together.

Taking a volume element $V$ out of the cylinder that contains $g$ molecules of $8 \mathrm{ASC} 10$ and $N_{\mathrm{w}} g$ molecules of water (strongly bound and bulk, $N_{\mathrm{w}} \approx 5.4$ ), we have $V=\pi R^{2} h=g v_{\mathrm{p}}+N_{\mathrm{w}} g v_{\mathrm{w}}$, where $R$ and $h$ are the radius and height of the element, $v_{\mathrm{p}}$ and $v_{\mathrm{w}}$ are the volume occupied by a surfactant headgroup $\left(367 \AA^{3}\right)$ and by a water molecule $\left(30 \AA^{3}\right)$, respectively (see Figure 13). The lateral surface of the cylindrical element is $A=2 \pi R h=$ $g a_{\mathrm{p}}+N_{\mathrm{w}} g a_{\mathrm{w}}$, where $a_{\mathrm{p}}$ and $a_{\mathrm{w}}$ are the cross-sections of the 8ASC10 headgroups and that of a water molecule (approximately $12 \AA^{2}$ ), respectively. From the two formulas we obtain

$$
a_{\mathrm{p}}=\frac{2\left(v_{\mathrm{p}}+N_{\mathrm{w}} v_{\mathrm{w}}\right)}{R}-N_{\mathrm{w}} a_{\mathrm{w}}
$$

which gives $a_{\mathrm{p}}=43 \AA^{2}$. If the cross-section of the surfactant headgroup with the surrounding water molecules is $a_{\mathrm{p}}+N_{\mathrm{w}} a_{\mathrm{w}}$ $=108 \AA^{2}$, the height of the single layer $(\tau)$ will be approximately $12 \AA$, from which we obtain that about seven to eight molecules

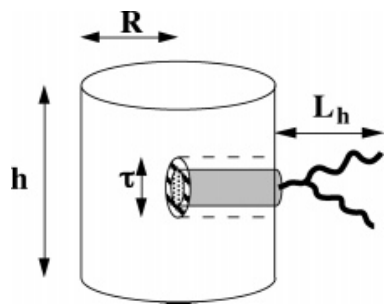

Figure 13. Volume element extracted from Figure 10. $R$ is the radius, $h$ the height, $\tau$ is the thickness of one single layer of molecules, and $L_{\mathrm{h}}$ the length of the surfactant hydrophobic tails. The gray region represents the 8ASC10 headgroup, and the dashed area contains the molecules of water surrounding the polar headgroups.

of surfactant occupy one single layer, as depicted in Figure 13. A similar calculation provides the average number of hydrocarbons closely associated to the surfactant hydrophobic chains $\left(N_{\mathrm{s}}\right)$. If $v_{\mathrm{s}}$ denotes the molecular volume of the hydrocarbon (obtained from its molecular mass and density at $25^{\circ} \mathrm{C}$ ), and $n$ is the number of surfactant molecules in one single layer, we have

$$
\pi\left[\left(R+L_{\mathrm{h}}\right)^{2}-R^{2}\right] \tau=N_{\mathrm{s}} v_{\mathrm{s}}+n v_{\mathrm{h}}
$$

from which $N_{\mathrm{s}}$ comes out to be about seven molecules for cyclohexane and four for $i$-octane per surfactant monomer. Finally, the calculation of the packing parameter for $8 \mathrm{ASC} 10$ results to be $p=v_{\mathrm{h}} /\left(L_{\mathrm{h}} a_{\mathrm{p}}\right)=1.1$ which corresponds to the formation of inverted micelles. ${ }^{35}$

Reducing Properties. The evaluation of the reducing activity of 8ASC10/cyclohexane and 8ASC10/water/cyclohexane samples according to the DPPH method indicated that the organogels possess a large antioxidant properties (above 90\%) even after 1 month from their preparation. This result is comparable to that of the native ascorbic acid and of its most common esters ASC $n$ and BOLA12 $2^{4,14}$ and confirms that self-assembled materials such as nanotubes and organogels obtained from vitamin $\mathrm{C}$ 
derivatives can be used for the solubilization and protection of molecules that are sensitive to the attack of free radicals. ${ }^{13}$

\section{Conclusions}

We report here the formation and properties of organogels containing a vitamin $\mathrm{C}$-based surfactant, 8ASC10, that bears two aliphatic chains linked to a L-ascorbic acid ring. The organogels are produced in the presence of hydrocarbons such as cyclohexane and $i$-octane, with or without water. The stability and behavior of the organogels have been studied at different temperatures and different compositions. DSC and SAXS experiments indicate that the organogels are formed by narrow rodlike structures with the polar headgroups and water molecules confined in the central hydrophilic pool, while the hydrophobic chains penetrate in the oil dispersing phase. Heating up the organogel above the sol-gel phase transition results in the formation of monodisperse inverted micellar systems. Rheology data indicate that the organogels are physically, not chemically, associated gels, stabilized by van der Waals forces and hydrogen bonding. The presence of a redox active polar head group in the surfactant adds an important and new functionality to the final organogels that can be used to solubilize and protect sensitive organic molecules.

Acknowledgment. The authors acknowledge the Consorzio Interuniversitario per lo Sviluppo dei Sistemi a Grande Interfase (CSGI, Florence) and the Ministero dell'Istruzione, dell'Università e della Ricerca (MIUR, Rome) for partial financial support.

\section{References and Notes}

(1) Nihro, Y.; Miyataka, H.; Sudo, T.; Matsumoto, H.; Sathoh, T. J. Med. Chem. 1991, 34, 2152-2157. 5670.

(2) Bisby, R. H.; Parker, A. W. J. Am. Chem. Soc. 1995, 117, 5664-

(3) Lo Nostro, P.; Capuzzi, G.; Romani, A.; Mulinacci, N. Langmuir 2000, 16, 1744-1750.

(4) Lo Nostro, P.; Capuzzi, G.; Pinelli, P.; Mulinacci, N.; Romani, A.; Vincieri, F. F. Colloids Surf., A 2000, 167, 83-93.

(5) Capuzzi, G.; Lo Nostro, P.; Kulkarni, K.; Fernandez, J. E.; Vincieri, F. F. Langmuir 1996, 12, 5413-5418.

(6) Capuzzi, G.; Lo Nostro, P.; Kulkarni, K.; Fernandez, J. E. Langmuir 1996, 12, 3957-3963.

(7) Capuzzi, G.; Kulkarni, K.; Fernandez, J. E.; Vincieri, F. F.; Lo Nostro, P. J. Colloid Interface Sci. 1997, 186, 271-279.
(8) Palma, S.; Manzo, R. H.; Allemandi, D.; Fratoni, L.; Lo Nostro, P. Langmuir 2002, 18, 9219-9224.

(9) Lo Nostro, P.; Ninham, B. W.; Fratoni, L.; Palma, S.; Manzo, R. H.; Allemandi, D.; Baglioni, P. Langmuir 2003, 19, 3222-3228.

(10) Lo Nostro, P.; Ninham, B. W.; Ambrosi, M.; Fratoni, L.; Palma, S.; Allemandi, D.; Baglioni, P. Langmuir 2003, 19, 9583-9591.

(11) Ambrosi, M.; Lo Nostro, P.; Fratoni, L.; Dei, L.; Ninham, B. W.; Palma, S.; Manzo, R. H.; Allemandi, D.; Baglioni, P. Phys. Chem. Chem. Phys. 2004, 6, 1401-1407.

(12) Palma, S.; Manzo, R. H.; Allemandi, D.; Fratoni, L.; Lo Nostro, P. J. Pharm. Sci. 2002, 91, 1810-1816.

(13) Bilia, A. R.; Bergonzi, M. C.; Vincieri, F. F.; Lo Nostro, P.; Morris, G. A. J. Pharm. Sci. 2002, 91, 2265-2270.

(14) Ambrosi, M.; Fratini, E.; Alfredsson, V.; Ninham, B. W.; Giorgi,

R.; Lo Nostro, P.; Baglioni, P. J. Am. Chem. Soc. 2006, 128, 7209-7214.

(15) Terech, P.; Weiss, R. G. Chem. Rev. 1997, 97, 3133-3159.

(16) Estroff, L. A.; Hamilton, A. D. Chem. Rev. 2004, 104, 1201-1217.

(17) George, M.; Weiss, R. G. Acc. Chem. Res. 2006, 39, 489-497.

(18) Blanton, T.; Huang, T. C.; Toraya, H.; Hubbard, C. R.; Robie, S. B.; Louer, D.; Gobel, H. E.; Will, G.; Gilles, R.; Raftery, T. Powder Diffr. 1995, 10, 91-95.

(19) Lake, J. A. Acta Crystallogr. 1967, 23, 191-194.

(20) Allinger, N. L. J. Am. Chem. Soc. 1977, 99, 8127-8134.

(21) Guinier, A.; Fournet, G. In Small-Angle Scattering of X-Rays; Wiley: New York, 1955.

(22) Evans, D. F.; Wennerström, H. In The Colloidal Domain, 2nd ed.; Wiley-VCH: New York, 1999.

(23) Evans, D. F.; Mitchell, D. J.; Ninham, B. W. J. Phys. Chem. 1986, 90, 2817-2825.

(24) Barnes, I. S.; Hyde, S. T.; Ninham, B. W.; Derian, P. J.; Drifford, M.; Zemb, T. N. J. Phys. Chem. 1988, 92, 2286-2293.

(25) Lisiecki, I.; André, P.; Filankembo, A.; Petit, C.; Tanori, J.; GulikKrzywicki, T.; Ninham, B. W.; Pileni, M. P. J. Phys. Chem. B 1999, 103, 9168-9175.

(26) Lisiecki, I.; André, P.; Filankembo, A.; Petit, C.; Tanori, J.; GulikKrzywicki, T.; Ninham, B. W.; Pileni, M. P. J. Phys. Chem. B 1999, 103, 9176-9189.

(27) André, P.; Ninham, B. W.; Pileni, M. P. Adv. Colloid Interface Sci. 2001, 89-90, 155-167.

(28) Abdallah, D. J.; Weiss, R. G. Adv. Mater. 2000, 12, 1237-1247.

(29) George, M.; Weiss, R. G. Chem. Mater. 2003, 15, 2879-2888.

(30) Angelico, R.; Ceglie, A.; Colafemmina, G.; Lopez, F.; Murgia, S.; Olsson, U.; Palazzo, G. Langmuir 2005, 21, 140-148.

(31) Shchipunov, Y. A. Colloids Surf., A 2001, 183-185, 541-554.

(32) Fuhrhop, J.-H.; Helfrich, W. Chem. Rev. 1993, 93, 1565-1582.

(33) Terech, P.; Coutin, A.; Giroud-Godquin, A. M. J. Phys. Chem. B 1997, 101, 6810-6818.

(34) Hyde, S. T.; Ninham, B. W.; Zemb, T. N. J. Phys. Chem. 1989, 93, 1464-1471.

(35) Mitchell, D. J.; Ninham, B. W. J. Chem. Soc., Faraday Trans. 2 1981, 77, 601-629. 